\title{
Preparation and quantification of radioactive particles for tracking hydrodynamic behavior in multiphase reactors
}

\begin{abstract}
Radioactive particle tracking (RPT) has emerged as a promising and versatile technique that can provide rich information about a variety of multiphase flow systems. However, RPT is not an off-the-shelf technique, and thus, users must customize RPT for their applications. This paper presents a simple procedure for preparing radioactive tracer particles created via irradiation with neutrons from the TRIGA Mark II research reactor. The present study focuses on the performance evaluation of encapsulated gold and scandium particles for applications as individual radioactive tracer particles using qualitative and quantitative neutron activation analysis (NAA) and an X-ray microcomputed tomography (X-ray MicroCT) scanner installed at the Malaysian Nuclear Agency.
\end{abstract}

Keyword: Radioactive particle tracking; Multiphase chemical reactors; Hydrodynamic behavior; Image reconstruction; Microcomputed tomography 